\section{Concentração de hemoglobina e anemia em crianças no Estado de Pernambuco, Brasil: fatores sócio-econômicos e de consumo alimentar associados}

\author{
Hemoglobin level and anemia in children in \\ the State of Pernambuco, Brazil: association with \\ socioeconomic and food consumption factors
}

Maria Alice Araújo Oliveira 1,2 Mônica Maria Osório ${ }^{1}$

Maria Cristina Falcão Raposo ${ }^{3}$

\author{
1 Departamento de Nutrição, \\ Universidade Federal de \\ Pernambuco, Recife, Brasil. \\ 2 Departamento de Nutrição, \\ Universidade Federal de \\ Alagoas, Maceió, Brasil. \\ 3 Departamento de \\ Estatística, Universidade \\ Federal de Pernambuco, \\ Recife, Brasil. \\ Correspondência \\ M. A. A. Oliveira \\ Programa de Pós-graduação \\ em Nutrição, Departamento \\ de Nutrição, Universidade \\ Federal de Pernambuco. \\ Av. Prof. Moraes Rego 1235, \\ Cidade Universitária \\ Recife, PE 50670-901, Brasil. \\ alicemcz@superig.com.br
}

\section{Abstract}

This study aimed to assess hemoglobin level and anemia prevalence and their association with food-consumption and socioeconomic variables. A cross-sectional survey was conducted in the State of Pernambuco, Brazil, in 1997, using threestage sampling which included 746 6-to-59month-old children. Hemoglobin testing and a 24-hour food recall interview were performed. The anemia rate was $40.6 \%$ and was higher in the rural area and inversely proportional to age, income, and maternal schooling. Most children (88.9\%) consumed cow's milk. Anemia was associated directly with the proportion of calories from cow's milk and indirectly with the iron level. Child's age, geographic area, per capita family income, maternal schooling, iron density (total, heme, and non-heme), and the proportion of calories from cow's milk in the diet were determinants for anemia.

Anemia; Risk Factors; Food Consumption; Bottle Feeding; Infant Nutrition

\section{Introdução}

A anemia ferropriva é o problema carencial de maior prevalência nos primeiros anos de vida, em todas as regiões do Brasil. Em crianças menores de cinco anos, as prevalências variam entre 31,4 e $47,8 \%$ 1. Numa abordagem sobre a transição nutricional no Brasil, Batista \& Rissin 2 apontaram para três aspectos importantes sobre a situação das anemias: o declínio da desnutrição não foi acompanhado por uma evolução favorável das anemias; não existem diferenças marcantes na ocorrência das anemias entre as diversas macrorregiões; e o problema afeta ricos e pobres. Outro aspecto de importância epidemiológica é a tendência temporal de aumento da prevalência da anemia nas crianças menores de cinco anos 2,3.

O estudo dos fatores associados ao aparecimento da anemia é de grande importância para a compreensão do problema. Entre os seus principais determinantes do problema encontram-se as precárias condições sócio-econômicas e ambientais, infecções, baixo peso ao nascer, desmame precoce e ingestão deficiente de alimentos ricos em ferro. Este último, entretanto, ainda merece maiores investigações 3 .

Em Pernambuco, Osório et al. ${ }^{4}$ verificaram uma alta prevalência de anemia em crianças de 6 a 59 meses de idade e um consumo alimentar de ferro inferior às recomendações diárias. Conseqüentemente, o ferro biodisponível encon- 
trava-se baixo, sendo sua quantidade diretamente proporcional à idade da criança. Os principais fatores explicativos para a variação da concentração de hemoglobina foram: idade da criança, ingestão de ferro biodisponível, retinol sérico, diarréia, tratamento da água, saneamento e baixo peso ao nascer 5 .

$\mathrm{O}$ aumento da anemia ferropriva em crianças pode ser decorrente das mudanças nos hábitos alimentares que acompanham a transição nutricional no país 3 . Também há indícios de que o alto consumo de leite de vaca, especialmente na alimentação infantil, pode estar associado à elevada prevalência de anemia 6 . A introdução desse alimento é precoce e a quantidade consumida é maior quanto menor a idade da criança, tendendo a diminuir à medida que outros alimentos não-lácteos são introduzidos na dieta, elevando-se o consumo de energia e ferro 6,7 .

Em virtude da gravidade da situação da anemia no Estado de Pernambuco, Brasil e, de maneira geral, do alto consumo de leite na alimentação infantil 6,8, o presente trabalho tem como objetivo avaliar a associação da concentração de hemoglobina e da prevalência de anemia com as variáveis sócio-econômicas, consumo de leite de vaca e outras variáveis relacionadas à dieta.

\section{Métodos}

Para a realização do presente estudo foi utilizado o banco de dados da II Pesquisa Estadual de Saúde e Nutrição ${ }^{9}$, realizada no período de 17 de fevereiro a 16 de maio de 1997 . Essa pesquisa teve como objetivo atualizar e ampliar o diagnóstico da situação de saúde, nutrição, alimentação e condições sócio-econômicas da população do Estado de Pernambuco, com ênfase no grupo materno-infantil. Para o dimensionamento da amostra utilizou-se a prevalência de 8,3\% de desnutrição em crianças do Nordeste, segundo o índice peso/idade, obtido na Pesquisa Nacional sobre Demografia e Saúde 10. A amostra aleatória foi sorteada em três estágios: municípios, setores censitários e domicílios. Para permitir a comparação dos resultados com a I Pesquisa Estadual de Saúde e Nutrição 11 foram considerados na seleção os mesmos $18 \mathrm{mu}$ nicípios dessa pesquisa, sorteados de forma aleatória, proporcional ao tamanho da população, ou seja, os municípios com maior população tiveram maior probabilidade de serem sorteados. Tal como na pesquisa anterior, definiuse previamente que seria investigado, em cada setor censitário, um total de 46 crianças. A par- tir dessa definição foi feito um sorteio aleatório simples dos setores censitários, considerando a proporcionalidade da população de cada município, resultando 45 setores censitários de um total de 2.655. Por último, com base no mapa censitário, foi selecionada aleatoriamente uma quadra e depois uma esquina dessa quadra, a partir da qual os domicílios foram visitados um a um, seguindo o sentido horário, identificando todas as crianças menores de cinco anos. Este procedimento garante a aleatoriedade das crianças sorteadas. No total, foram estudadas 2.078 crianças, das quais aproximadamente um terço foi selecionado sistematicamente (uma a cada duas crianças), para a realização do inquérito de consumo alimentar e dosagem de hemoglobina, totalizando 992 crianças. Destas, 24 foram retiradas do estudo por inconsistência nos questionários de consumo alimentar e 222 menores de seis meses que não tiveram o sangue coletado para a dosagem de hemoglobina. A amostra estudada foi de 746 crianças de 6 a 59 meses.

A dosagem de hemoglobina foi realizada em amostras de sangue, obtidas mediante punção venosa, utilizando-se o equipamento Hemocue, de leitura imediata. Foram consideradas anêmicas as crianças com concentração de hemoglobina inferior a $11 \mathrm{~g} / \mathrm{dL}$, de acordo com o critério adotado pela Organização Mundial da Saúde 12. A prevalência da anemia foi estimada usando-se um fator de correção, definido em função do procedimento amostral e da população do Estado de Pernambuco, estimada pelo Instituto Brasileiro de Geografia e Estatística 13 .

O método utilizado para a obtenção dos dados referentes ao consumo alimentar das crianças menores de cinco anos foi o recordatório das últimas 24 horas. Foi realizada entrevista domiciliar com a mãe biológica ou adotiva, ou com o indivíduo adulto responsável pelo cuidado da criança, utilizando-se um formulário, no qual se anotavam o horário das refeições, os alimentos consumidos, formas de preparo e marcas comerciais (alimentos industrializados), as quantidades preparadas, oferecidas e consumidas pela criança, em medidas caseiras e medidas de volume. Foram considerados os alimentos consumidos nas diversas refeições diárias (desjejum, lanches, almoço, jantar e ceia), e as quantidades referidas em medidas caseiras foram posteriormente convertidas em peso líquido, permitindo, assim, uma análise qualitativa e quantitativa da dieta.

Com as informações referentes ao consumo alimentar foi construído o banco de dados, utilizando-se o programa Virtual Nutri 14 para aná- 
lise da composição nutricional dos alimentos em macronutrientes e micronutrientes, sendo aqueles não encontrados nesse programa, inseridos a partir de tabelas de composição química de alimentos 15,16,17. A ingestão de leite materno foi estimada baseando-se no número de mamadas, considerando o volume de acordo com a idade da criança 14 .

O cálculo da densidade da dieta em ferro foi efetuado mediante a totalização da quantidade de ferro (mg) ingerida pela criança, dividindo-se esse valor pelo somatório de calorias ingeridas pela criança no dia, multiplicando-se o resultado por mil. A densidade da dieta em ferro total, ferro heme e ferro não-heme foi expressa, no presente estudo, em mg de ferro para cada $1.000 \mathrm{kcal}$ da dieta.

O ferro heme na dieta foi representado pela quantidade total diária de ferro proveniente do consumo de carnes, frangos e peixes, e o ferro não-heme foi obtido totalizando-se a quantidade de ferro proveniente dos demais alimentos, tal como utilizado no estudo de Levy-Costa \& Monteiro 6 .

O consumo diário de leite foi considerado em ml de leite fluido (in natura, pasteurizado e UHT ou comercialmente estéril). Quando foi referido o consumo de leite em pó, a quantidade informada (em g) foi transformada em equivalente de leite fluido $(\mathrm{em} \mathrm{ml})$. Adotou-se o valor de diluição de $12,93 \%$, obtido da média das diluições calculada com base em uma subamostra de 82 crianças, correspondente a $20 \%$ daquelas que consumiam leite em pó, sorteada aleatoriamente. A proporção de calorias de leite na dieta foi obtida dividindo-se o total de calorias (kcal) proveniente do consumo de leite pelas calorias totais da dieta consumidas pela criança no dia.

Verificou-se a associação da concentração de hemoglobina e da prevalência da anemia com as variáveis independentes: idade da criança (em meses), sexo, escolaridade materna (em anos de estudo), áreas geográficas (Região Metropolitana do Recife, interior urbano e interior rural), renda familiar per capita (em salários mínimos), consumo e proporção de calorias do leite de vaca, ferro total, ferro heme, ferro nãoheme, densidade de ferro total, de ferro heme e de ferro não-heme.

Para as análises estatísticas foi utilizado o programa SPSS, versão 7.5 (SPSS Inc., Chicago, Estados Unidos). Inicialmente foi verificado o comportamento das variáveis quanto à consistência e observada sua distribuição de freqüência mediante a aplicação do teste de Kolmogorov-Smirnov. Foram calculadas medidas de tendência central (média) e de dispersão (desvio padrão). Foram elaboradas tabelas de contingência para prevalências de anemia e calculadas médias de concentração de hemoglobina, segundo categorias de variáveis sócio-econômicas, de consumo alimentar (volume de leite, proporção de calorias do leite e densidade de ferro) e tipo de leite consumido. No estudo da associação entre a ocorrência de anemia e variáveis de natureza categórica (área geográfica) ou categorizadas, foi utilizado o teste qui-quadrado de associação de Pearson e, no caso das categorias apresentarem uma variação de ordem (renda, escolaridade e idade), foi utilizado o teste $\chi^{2}$ para tendência. Na comparação das médias de concentração de hemoglobina foi utilizado o teste t de comparação de médias quando a variável apresentava duas categorias. Para as variáveis com mais de duas categorias que apresentaram distribuição normal (hemoglobina e proporção de calorias provenientes do leite) foi utilizado o teste de comparação de médias ANOVA e, quando houve diferença estatisticamente significante, foi realizado o teste de Tukey para identificar quais as categorias que diferiram entre si. Para as variáveis relacionadas à dieta que apresentaram distribuição assimétrica foi utilizado o teste não paramétrico de Kruskal Wallis (para mais de dois grupos não emparelhados). Em todas as análises foi considerado o nível de significância $\mathrm{p}<0,05$.

A II Pesquisa Estadual de Saúde e Nutrição atendeu às Normas Regulamentares de Pesquisas Envolvendo Seres Humanos - Resolução 196/96, do Conselho Nacional de Saúde, e foi aprovada pelo Comitê de Ética em Pesquisa do Instituto Materno Infantil de Pernambuco. As crianças diagnosticadas como anêmicas receberam tratamento com 3mg de ferro elementar (como sulfato ferroso) por quilo de peso, semanalmente, durante seis meses.

\section{Resultados}

A Tabela 1 apresenta a concentração de hemoglobina e a prevalência de anemia de acordo com sexo, idade e variáveis sócio-econômicas. A concentração média de hemoglobina das crianças estudadas foi de $10,9 \pm 1,6 \mathrm{~g} / \mathrm{dL}$, havendo diferenças estatisticamente significantes entre as categorias de idade, apresentandose menor nas duas primeiras ( $<24$ meses) que, por sua vez, diferem das demais (Tukey $\mathrm{p}<0,05$ ). Com relação às categorias de renda per capita familiar, verifica-se que houve diferença significante: as três primeiras categorias são semelhantes (<1 salário mínimo) e diferem da última (Tukey p $<0,05)$. A hemoglobina apresen- 
Distribuição da concentração de hemoglobina e prevalência de anemia segundo sexo, idade, indicadores sócio-econômicos e áreas geográficas, em crianças de 6 a 59 meses de idade. Estado de Pernambuco, Brasil, 1997.

\begin{tabular}{|c|c|c|c|c|c|}
\hline \multirow[t]{2}{*}{ Variáveis } & \multirow[b]{2}{*}{$\mathrm{n}$} & \multicolumn{2}{|c|}{ Hemoglobina (g/dl) } & \multicolumn{2}{|c|}{ Prevalência de anemia } \\
\hline & & Média & Desvio padrão & $\%$ & $\mathrm{n}$ \\
\hline \multicolumn{6}{|l|}{ Sexo } \\
\hline Masculino & 380 & 11,0 & 1,6 & 45,3 & 172 \\
\hline Feminino & 366 & 10,9 & 1,5 & 47,8 & 175 \\
\hline 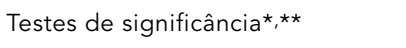 & & $\mathrm{T}=1,15$ & $p=0,25$ & $\chi^{2}=0,49$ & $p=0,485$ \\
\hline \multicolumn{6}{|l|}{ Idade (meses) } \\
\hline $06 \vdash 12$ & 130 & 10,1 & 1,5 & 68,5 & 89 \\
\hline $12 \vdash 24$ & 232 & 10,5 & 1,5 & 59,1 & 137 \\
\hline $24+36$ & 145 & 11,0 & 1,5 & 43,4 & 63 \\
\hline $36+48$ & 129 & 11,7 & 1,3 & 23,3 & 30 \\
\hline$\geq 48$ & 110 & 11,7 & 1,3 & 25,5 & 28 \\
\hline Testes de significância ${ }^{\star \star \star, \# ~}$ & & $F=32,72$ & $p=0,000$ & $\chi^{2}($ trend $)=81,92$ & $p=0,000$ \\
\hline \multicolumn{6}{|l|}{$\begin{array}{l}\text { Renda familiar per capita } \\
\text { (salários mínimos) }\end{array}$} \\
\hline$<0,25$ & 229 & 10,7 & 1,6 & 52,8 & 121 \\
\hline $0,25 \vdash 0,50$ & 202 & 10,8 & 1,6 & 51,0 & 103 \\
\hline $0,50 \vdash 1,0$ & 151 & 11,1 & 1,5 & 41,1 & 62 \\
\hline$\geq 1,00$ & 156 & 11,3 & 1,4 & 36,5 & 57 \\
\hline 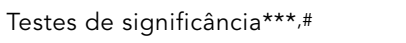 & & $F=5,91$ & $p=0,000$ & $\chi^{2}($ trend $)=12,82$ & $p=0,000$ \\
\hline \multicolumn{6}{|l|}{$\begin{array}{l}\text { Escolaridade materna } \\
\text { (anos de estudo) }\end{array}$} \\
\hline $0 \vdash 4$ & 312 & 10,6 & 1,6 & 54,5 & 170 \\
\hline $4 \vdash 8$ & 249 & 11,0 & 1,5 & 45,8 & 114 \\
\hline $8 \vdash 11$ & 75 & 11,4 & 1,6 & 37,3 & 28 \\
\hline$\geq 11$ & 109 & 11,4 & 1,3 & 32,1 & 35 \\
\hline 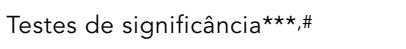 & & $F=9,70$ & $p=0,000$ & $\chi^{2}($ trend $)=19,46$ & $p=0,000$ \\
\hline \multicolumn{6}{|l|}{ Áreas geográficas } \\
\hline Região Metropolitana do Recife & 259 & 11,0 & 1,6 & 44,0 & 114 \\
\hline Interior urbano & 242 & 11,2 & 1,4 & 40,1 & 97 \\
\hline Interior rural & 245 & 10,6 & 1,6 & 55,5 & 136 \\
\hline 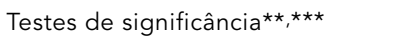 & & $F=9,16$ & $p=0,000$ & $\chi^{2}=12,64$ & $p=0,002$ \\
\hline Total & 746 & 10,9 & 1,6 & 40,6 & 347 \\
\hline
\end{tabular}

* Teste t para comparação de médias:

** Teste do qui-quadrado de associação de Pearson $\left(\chi^{2}\right)$ $\star * \star$ ANOVA

\# Teste do qui-quadrado para tendência linear ( $\chi^{2}$ trend).

tou-se mais baixa nas crianças cujas mães encontravam-se na faixa de menor escolaridade ( $<4$ anos de estudo) e diferiu significantemente das demais (Tukey $\mathrm{p}<0,05$ ). As crianças do interior rural apresentaram menor concentração de hemoglobina, diferindo das outras duas áreas geográficas (Tukey $\mathrm{p}<0,05$ ).

A prevalência de anemia para o Estado de Pernambuco foi de $40,6 \%$ e apresentou tendência de diminuição, estatisticamente significan- te, com o aumento da idade, renda familiar per capita e escolaridade materna, mostrando-se mais acentuada entre as crianças do Interior Rural (55,5\%).

A Tabela 2 apresenta a prevalência de anemia e a concentração de hemoglobina de acordo com o tipo de leite consumido. Verificou-se que entre as crianças estudadas predominava o consumo de leite de vaca $(88,9 \%)$, especialmente o leite em pó integral $(47,8 \%)$ e leite flui- 
Distribuição da concentração de hemoglobina e prevalência de anemia segundo o tipo de leite consumido, em crianças de 6 a 59 meses de idade. Estado de Pernambuco, Brasil, 1997.

\begin{tabular}{|c|c|c|c|c|c|c|}
\hline \multirow[t]{2}{*}{ Tipo de leite } & \multirow[b]{2}{*}{$\mathrm{n}$} & \multirow[b]{2}{*}{$\%$} & \multicolumn{2}{|c|}{ Hemoglobina (g/dl) } & \multicolumn{2}{|c|}{ Prevalência de anemia } \\
\hline & & & Média & desvio padrão & $\mathrm{n}$ & $\%$ \\
\hline Leite materno & 29 & 3,9 & 10,4 & 1,3 & 18 & 62,1 \\
\hline Leite fluido & 180 & 24,1 & 10,9 & 1,6 & 86 & 47,8 \\
\hline Leite em pó integral & 357 & 47,8 & 10,9 & 1,6 & 167 & 46,8 \\
\hline Leite em pó modificado & 56 & 7,5 & 10,9 & 1,3 & 24 & 42,9 \\
\hline Leite materno + outros leites & 42 & 5,6 & 10,5 & 1,5 & 27 & 64,3 \\
\hline Leite fluido + outros leites em pó & 28 & 3,8 & 11,5 & 1,7 & 11 & 39,3 \\
\hline Sem consumo de leite & 54 & 7,2 & 11,6 & 1,3 & 14 & 25,9 \\
\hline Teste de significância*,** & & & $t=3,17$ & $p=0,004$ & $\chi^{2}=18,37$ & $p=0,005$ \\
\hline \multicolumn{7}{|l|}{ Consumo de leite de vaca } \\
\hline Sim & 663 & 88,9 & 10,9 & 1,6 & 315 & 47,5 \\
\hline Não & 83 & 11,1 & 11,2 & 1,4 & 32 & 38,6 \\
\hline Testes de significância* & & & $t=1,59$ & $p=0,11$ & $\chi^{2}=2,38$ & $p=0,12$ \\
\hline
\end{tabular}

* Teste t;

** Teste do qui-quadrado $\left(\chi^{2}\right)$.

do $(24,1 \%)$. Apenas $7,5 \%$ das crianças consumiam leite modificado ou fórmula infantil e $3,9 \%$ permaneciam com o leite materno como único tipo de leite. Houve diferença estatisticamente significante entre os diversos tipos de leite, a prevalência da anemia e a concentração de hemoglobina.

As crianças sem consumo de leite apresentaram menor prevalência de anemia $(25,9 \%)$ e maior média de concentração de hemoglobina $(11,6 \mathrm{~g} / \mathrm{dl})$, sendo diferente das crianças que consumiam leite materno, leite em pó (integral e modificado) e materno mais outros tipos de leite (Tukey, $\mathrm{p}<0,05)$. Ao serem agrupadas em duas categorias (consumiu ou não leite de vaca), verifica-se que não houve diferença estatística significante entre a variável consumo de leite de vaca e as médias de hemoglobina e prevalência de anemia.

A Tabela 3 apresenta o resultado da análise da concentração de hemoglobina e prevalência de anemia com as variáveis: consumo diário de leite e proporção de calorias do leite, densidade de ferro (total) e ferro não-heme em quartis e densidade de ferro heme em duas categorias, separadas pela mediana. Houve diferença estatisticamente significante entre a concentração média de hemoglobina e a proporção de calorias do leite e densidade de ferro (total, heme e não-heme), porém não ocorreu diferença com o volume de leite. A prevalência de anemia apresentou tendência linear significante de aumento à medida que se elevou a proporção de calorias do leite na dieta, e de redução com o au- mento da densidade de ferro (total, heme e não-heme).

\section{Discussão}

As prevalências de anemia encontradas neste estudo corroboram com os altos porcentuais encontrados no país, especialmente entre as crianças menores de 24 meses 18,19,20,21. Durante os seis primeiros meses de vida, as crianças nascidas a termo, alimentadas com leite materno exclusivo, suprem suas necessidades a partir de suas reservas e da alta biodisponibilidade do ferro desse leite. A partir dessa idade, o leite materno já não é suficiente, sendo necessária a introdução de outros alimentos complementares ricos em ferro 17,22,23. A maior prevalência de anemia nas crianças menores provavelmente está associada às necessidades elevadas de ferro para fazer face ao crescimento intenso, ao mesmo tempo em que a sua dieta é pobre em alimentos fontes de ferro, especialmente do ferro biodisponível, sendo baseada no consumo de leite de vaca.

Para suprir as necessidades de ferro das crianças menores, é necessário que sua dieta contenha mais ferro por unidade de energia do que a dieta das crianças de maior idade 23 . No entanto, sabe-se que, no Nordeste, mesmo quando disponíveis para a família, alimentos como carnes, peixes, vísceras (fontes de ferro), além de frutas e vegetais (fontes de vitamina $\mathrm{C}$, estimuladores da absorção de ferro) não são con- 
Distribuição da concentração de hemoglobina e prevalência de anemia segundo o volume de leite consumido, proporção de calorias do leite, densidade de ferro total, de ferro heme e de ferro não-heme, em crianças de 6 a 59 meses de idade. Estado de Pernambuco, Brasil, 1997.

\begin{tabular}{|c|c|c|c|c|}
\hline & \multicolumn{2}{|c|}{ Hemoglobina (g/dl) } & \multicolumn{2}{|c|}{ Prevalência de anemia } \\
\hline & Média & Desvio padrão & $\mathrm{n}$ & $\%$ \\
\hline \multicolumn{5}{|l|}{ Volume de leite (ml) } \\
\hline $0 \vdash 210,0$ & 11,1 & 1,4 & 77 & 41,4 \\
\hline $210,0 \vdash 464,4$ & 10,9 & 1,7 & 81 & 43,3 \\
\hline $464,4 \vdash 789,6$ & 10,8 & 1,6 & 97 & 52,2 \\
\hline$\geq 789,6$ & 10,8 & 1,6 & 92 & 49,2 \\
\hline Teste de significância*,** & $F=1,38$ & $p=0,25$ & $\chi^{2}($ trend $)=3,38$ & $p=0,06$ \\
\hline \multicolumn{5}{|l|}{ Calorias do leite (\%) } \\
\hline $0 \vdash 13,0$ & 11,2 & 1,3 & 68 & 36,4 \\
\hline $13,0 \vdash 27,6$ & 11,2 & 1,6 & 68 & 36,6 \\
\hline $27,6 \vdash 42,8$ & 10,6 & 1,6 & 107 & 57,2 \\
\hline $42,8 \vdash 90,8$ & 10,5 & 1,6 & 104 & 55,9 \\
\hline 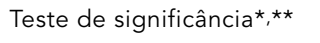 & $F=11,98$ & $p=0,000$ & $\chi^{2}($ trend $)=23,55$ & $p=0,000$ \\
\hline \multicolumn{5}{|c|}{ Densidade de ferro total (mg/1.000kcal) } \\
\hline $0 \vdash 3,3$ & 10,5 & 1,6 & 108 & 57,4 \\
\hline $3,3 \vdash 5,2$ & 10,9 & 1,5 & 87 & 47,0 \\
\hline $5,2 \vdash 7,5$ & 11,2 & 1,5 & 76 & 40,6 \\
\hline $7,5+20,0$ & 11,0 & 1,5 & 76 & 40,9 \\
\hline Teste de significância*,** & $F=7,56$ & $p=0,000$ & $\chi^{2}($ trend $)=11,87$ & $p=0,000$ \\
\hline \multicolumn{5}{|c|}{ Densidade de ferro não-heme (mg/1.000kcal) } \\
\hline $0 \vdash 2,6$ & 10,6 & 1,6 & 99 & 52,9 \\
\hline $2,6 \vdash 4,1$ & 10,9 & 1,5 & 95 & 51,1 \\
\hline $4,1 \vdash 6,2$ & 11,2 & 1,5 & 73 & 38,6 \\
\hline $6,2 \vdash 20,0$ & 11,0 & 1,6 & 80 & 43,5 \\
\hline 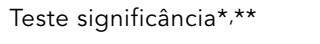 & $F=3,36$ & $p=0,018$ & $\chi^{2}($ trend $)=6,28$ & $p=0,012$ \\
\hline \multicolumn{5}{|c|}{ Densidade de ferro heme $(\mathrm{mg} / 1.000 \mathrm{kcal})$} \\
\hline $0 \vdash 0,04$ & 10,5 & 1,5 & 205 & 55,9 \\
\hline$\geq 0,04$ & 11,3 & 1,5 & 142 & 37,5 \\
\hline Teste de significância ${ }^{\star \star *}, \#$ & $\mathrm{~T}=7,10$ & $p=0,000$ & $\chi^{2}=25,35$ & $p=0,000$ \\
\hline
\end{tabular}

sumidos pelas crianças menores de dois anos. A partir de 24 meses, as crianças consomem menor quantidade de leite de vaca e ingerem alimentos fontes de ferro que fazem parte dos hábitos alimentares da família 24,25,26.

Em relação aos indicadores sócio-econômicos, outros estudos também detectaram associação entre anemia e renda e entre anemia e escolaridade 3,19,21. Analisando-se dois estudos na cidade de São Paulo (1984-1996), Monteiro et al. 3 , ressaltam a tendência de aumento da anemia, de forma mais desfavorável nos estra- tos de renda inferior e intermediário. A escolaridade materna está associada à renda, uma vez que um maior nível de instrução pode proporcionar maiores chances de emprego, melhores salários e, portanto, maior poder aquisitivo e melhor acesso aos alimentos. Além disso, pode condicionar uma melhor capacidade da família em alocar racionalmente a renda e influenciar os cuidados com a saúde e alimentação da criança 3,27 .

O fato de o interior rural apresentar maior prevalência de anemia reflete as precárias con- 
dições de vida a que estavam submetidas as famílias das crianças, uma vez que $85,5 \%$ dos domicílios não possuíam canalização interna de água tratada, apenas $30 \%$ tinham fossas sépticas, havendo predomínio de renda per capita menor do que $1 / 4$ do salário mínimo (39,6\%), com uma média de escolaridade materna de 3,8 anos 9. A escassez, a dificuldade de acesso aos alimentos e a falta de conhecimento do seu valor nutritivo podem determinar um consumo inadequado.

$\mathrm{O}$ aleitamento materno exclusivo é recomendado até os seis meses de vida, devendo ser continuado até os dois anos de idade, juntamente com alimentos complementares nutricionalmente adequados e seguros 23. Mesmo considerando que a faixa etária das crianças estudadas foi de 6 a 59 meses, identifica-se que apenas $3,9 \%$ delas consumiram leite materno, o que é muito pouco, uma vez que o percentual de menores de dois anos na amostra foi de $49 \%$. Essa situação era esperada, uma vez que a duração mediana do aleitamento materno para o Estado de Pernambuco, detectada na população estudada, foi de apenas 106 dias 9 .

O principal tipo de leite consumido pelas crianças foi o de vaca, especialmente o leite em pó integral, sem adição de ferro, corroborando com os achados de outros estudos 18,25,26. Mesmo em crianças menores de seis meses, a freqüência do consumo de leite de vaca nessa população foi mais elevada do que a do leite materno, e outros alimentos foram introduzidos tardiamente na alimentação da criança 25.

Estudos recentes identificaram que, em crianças menores de seis meses, o aleitamento materno exclusivo tinha efeito benéfico por manter níveis mais elevados de hemoglobina e que o consumo de leite de vaca, de forma exclusiva (aleitamento artificial) ou mesmo juntamente com o leite materno (aleitamento misto), reduzia os níveis de hemoglobina 28,29.

Quanto mais precoce é a introdução de leite de vaca, maior a chance de desenvolvimento da deficiência de ferro 29 . A caseína e as proteínas do soro de leite de vaca podem ser responsáveis pela baixa biodisponibilidade do ferro em fórmulas infantis 30 . O cálcio também pode inibir a absorção de ferro, e o consumo de leite ou queijo, junto às refeições fontes de ferro, reduz a absorção de ferro não-heme, razão pela qual se recomenda que os mesmos não sejam consumidos regularmente nas principais refeições fontes de ferro ${ }^{31}$. Também existem indícios de que o consumo de leite de vaca fresco (integral pasteurizado e homogeneizado) pode provocar microcitose, ferropenia e menor concentração de hemoglobina, além de diminui- ção da concentração de ferritina e perda de sangue oculto nas fezes 32,33 .

O fato de as crianças que não consumiam leite apresentarem menor prevalência de anemia e maior nível de hemoglobina, provavelmente ocorreu devido à maioria delas (94\%) terem idade maior ou igual a 24 meses e que não ingerindo leite, conseqüentemente, consumiam uma maior quantidade de alimentos fontes de ferro.

Em algumas situações, $\mathrm{o}$ aporte de calorias da dieta pode estar adequado, porém as necessidades de ferro não estão sendo supridas, o que indica que o problema não está na quantidade total de alimentos oferecidos à criança. Para avaliar a composição do ferro dietético, é necessário trabalhar com o consumo de ferro total, de ferro heme e de ferro não-heme e, especialmente, com suas densidades na dieta. A densidade de ferro pode ser considerada um bom indicador da qualidade da dieta em relação ao ferro, uma vez que relaciona o consumo desse micronutriente, ou de suas frações ferro heme e ferro não-heme às calorias da dieta, identificando se o problema está na composição da mesma. Esse indicador é particularmente importante na avaliação da dieta infantil, tendo em vista as suas elevadas necessidades de ferro 23 .

A importância dos indicadores do consumo alimentar foi demonstrada no presente estudo por meio da associação altamente significante das variáveis densidade de ferro, de ferro heme e de ferro não-heme com a concentração de hemoglobina. Monteiro et al. 3 consideram esse indicador como fator decisivo para aumentar ou diminuir o risco de anemia. Os autores fizeram uma estimativa de recomendação média diária de 8,0mg Fe/1.000kcal para crianças de 6 a 59 meses e verificaram que, entre 1984/1985 e 1995/1996, a densidade de ferro na dieta não se modificou $(5,4 \mathrm{mg} \mathrm{Fe} / 1.000 \mathrm{kcal})$, o que poderia justificar a manutenção da alta prevalência de anemia na cidade de São Paulo, porém, não explica o seu aumento.

O aumento da participação do leite de vaca nas calorias totais da dieta esteve associado ao aumento da prevalência de anemia e à diminuição da concentração de hemoglobina, resultados semelhantes aos encontrados no Município de São Paulo 6 . Esses achados apontam para uma possível relação entre o consumo de leite de vaca e a anemia em crianças, porém não são suficientes para explicá-la, exigindo um maior aprofundamento analítico dos resultados.

Freqüentemente, o leite, adicionado ou não de farinhas ou frutas, é utilizado como substituto ou complemento de refeições de crianças 
menores de dois anos 8,24. O leite de vaca in $n a$ tura, pasteurizado ou em pó integral, além de ser pobre em ferro, apresenta esse elemento sob a forma de ferro não-heme e possui baixa concentração de vitamina C. Se outros alimentos complementares ricos nesses micronutrientes não são oferecidos ou o são em quantidades limitadas e se o leite contribui com uma grande parte das calorias da dieta das crianças, torna-se difícil suprir as necessidades de ferro nessa faixa etária em virtude da baixa densidade de ferro desse alimento.

Deve-se salientar que existem limitações no presente estudo, visto que o método utilizado para inquérito alimentar (recordatório de 24 horas) representa apenas uma estimativa da realidade do consumo alimentar e que somente uma tomada não seria suficiente para mostrar a variabilidade intrapessoal, principalmente no que se refere a micronutrientes. Por outro lado, erros de estimativa são diminuídos quando se trabalha com amostras relativamente grandes, caso deste estudo.

Os resultados obtidos no presente estudo permitem concluir que a anemia é um problema grave no grupo estudado, principalmente no interior rural e entre as crianças mais novas. A baixa renda e a baixa escolaridade materna estiveram associadas à menor concentração de hemoglobina e à maior prevalência de anemia. O estudo da composição da dieta é de extrema importância para a identificação dos fatores de risco para o desenvolvimento desse problema nutricional e, conseqüentemente, para o planejamento de intervenções. Entre as variáveis relacionadas à dieta observou-se importante associação entre a densidade de ferro total, ferro heme e ferro não-heme, bem como a proporção de calorias do leite e a anemia. De uma forma geral, os estudos não se referem ao leite de vaca como um determinante da anemia na infância, porém, no presente estudo, ele foi o principal tipo de leite consumido pelas crianças e quanto maior a sua contribuição nas calorias totais, menor a densidade de ferro da dieta e maior a gravidade do problema. Os resultados evidenciam a necessidade de aprofundar estudos que enfoquem o papel da dieta (consumo de leite de vaca, densidade de ferro) e anemia.

Com base na situação aqui encontrada, devem ser consideradas ações preventivas de incentivo ao aleitamento materno exclusivo até os seis meses de idade e o seu prolongamento pelo menos até os dois anos. É importante que as mães sejam orientadas também sobre a alimentação complementar adequada rica em ferro e facilitadores da sua absorção, utilizando alimentos regionais que fazem parte do hábito alimentar da família, enfatizando a combinação de alimentos ricos em vitamina $C$ com aqueles que são fontes de ferro, evitando oferecer leite junto a estes últimos. A fortificação de alimentos infantis com ferro também se torna medida relevante para a prevenção da anemia e de suas conseqüências na infância. Outras ações para o combate ao problema são a suplementação medicamentosa (já contemplada no programa de intervenção e controle da deficiência de ferro do Ministério da Saúde), o controle das infecções parasitárias e a fortificação de alimentos com ferro.

Essas ações devem ser realizadas em conjunto com outras medidas, como o investimento na formação dos profissionais que atuam na rede de atenção básica à saúde, no sentido de melhorar a assistência pré-natal, prevenir a desnutrição materna e o baixo peso ao nascer. Ressalta-se, também, que políticas públicas de aumento da oferta de emprego (gerando aumento de renda) e escolarização devem ser implementadas, uma vez que contribuem de forma significativa para a diminuição da prevalência de anemia em crianças, principalmente naquelas residentes no Interior Rural.

\section{Resumo}

Este estudo teve como objetivo avaliar a associação entre a concentração de hemoglobina e prevalência de anemia com variáveis sócio-econômicas e de consumo alimentar. O estudo seccional, realizado em Pernambuco, Brasil, no ano 1997, utilizou a amostragem em três estágios. A amostra compreendeu 746 crianças de 6-59 meses, nas quais foram realizados dosagem de hemoglobina e inquérito dietético recordatório de 24 horas. A prevalência de anemia foi de 40,6\%, sendo maior no Interior Rural e diminuindo com o aumento da idade, da renda e da escolaridade materna. A maioria das crianças (88,9\%) consumia leite de vaca.
A anemia apresentou tendência de aumento com a elevação da proporção de calorias do leite e de redução com o aumento da densidade de ferro (total, heme e não-heme). A idade da criança, a área geográfica, a renda familiar per capita, a escolaridade materna, a densidade de ferro e a proporção de calorias do leite de vaca na dieta são fatores determinantes da anemia no grupo estudado.

Anemia; Fatores de Risco; Consumo de Alimentos; Alimentação Artificial; Nutrição Infantil 


\section{Colaboradores}

M. A. A. Oliveira e M. M. Osório participaram da concepção da pesquisa, revisão da literatura, processamento e análise dos dados e redação do artigo. M. C. F. Raposo participou da análise estatística dos dados e redação do artigo.

\section{Referências}

1. Santos L. Bibliografia sobre deficiência de micronutrientes no Brasil, 1990-2000: anemia. v. 2(a). Brasília: Organização Pan-Americana da Saúde/ Organização Mundial da Saúde; 2002.

2. Batista Filho M, Rissin A. A transição nutricional no Brasil: tendências regionais e temporais. Cad Saúde Pública 2003; 19 Suppl 1:S181-91.

3. Monteiro CA, Szarfarc SC, Mondini L. Tendência secular da anemia na infância na Cidade de São Paulo (1984-1996). Rev Saúde Pública 2000; 34 Suppl 6:62-72.

4. Osório MM, Lira PIC, Batista-Filho M, Ashworth A. Prevalence of anemia in children 6-59 months old in the state of Pernambuco, Brazil. Pan Am J Public Health 2001; 10:101-7.

5. Osório MM, Lira PIC, Ashworth A. Factors associated with $\mathrm{Hb}$ concentration in children aged 6-59 months in the state of Pernambuco, Brazil. Br J Nutr 2004; 91:307-14.

6. Levy-Costa RB, Monteiro CA. Consumo de leite de vaca e anemia na infância no Município de São Paulo; Rev Saúde Pública 2004; 38:797-803.

7. Souza SB, Szarfarc SC, Monteiro CA. Anemia e alimentação no primeiro ano de vida em relação ao aleitamento materno. Rev Saúde Pública 1997; 31:15-20.

8. Souza SB, Szarfarc SC, Souza JMP. Prática alimentar no primeiro ano de vida, em crianças atendidas nos centros de saúde escola do Município de São Paulo. Rev Nutr 1999; 12:167-74.
9. Ministério da Saúde. II Pesquisa Estadual de Saúde e Nutrição: saúde, nutrição, alimentação e condições sócio-econômicas no Estado de Pernambuco. Recife: Instituto Nacional de Alimentação e Nutrição; 1998.

10. Sociedade Civil do Bem-estar Familiar no Brasil. Pesquisa Nacional sobre Demografia e Saúde, 1996. Rio de Janeiro: Sociedade Civil do Bem-estar Familiar no Brasil; 1997.

11. Governo do Estado de Pernambuco/Fundo das Nações Unidas para a Infância. Crianças e adolescentes em Pernambuco: saúde, educação e trabalho. Brasília: Fundo das Nações Unidas para a Infância; 1992.

12. World Health Organization. Iron deficiency anaemia assessment, prevention and control: a guide for programme managers. Geneva: World Health Organization; 2001.

13. Instituto Brasileiro de Geografia e Estatística. Contagem nacional da população, 1996. Sistema IBGE de Recuperação Automática. http: / / www. sidra.ibge.gov.br (acessado em 08/Mar/2001).

14. Philippi ST, Szarfarc SC, Latterza AR. Virtual Nutri [programa de computador]. Versão 1.0 for Windows. São Paulo: Departamento de Nutrição. Faculdade de Saúde Pública da Universidade de São Paulo; 1996.

15. Instituto Brasileiro de Geografia e Estatística. Tabelas de composição de alimentos (Estudo Nacional da Despesa Familiar). Rio de Janeiro: Instituto Brasileiro de Geografia e Estatística; 1977. 
16. Leung WTW, Flores M. Tabla de composición de alimentos para uso en América Latina. Guatemala: Instituto de Nutrición de Centro America y Panamá; 1970.

17. Coordenação Geral de Políticas de Alimentação e Nutrição, Secretaria de Políticas de Saúde, Ministério da Saúde. Alimentos regionais brasileiros. Brasília: Ministério da Saúde; 2002. (Série F: Comunicação e Educação em Saúde).

18. Sichieri R, Szarfarc SC, Monteiro CA. Relação entre dieta e ocorrência de anemia ferropriva em crianças. J Pediatr (Rio J) 1988; 64:169-74.

19. Silva LSM, Giugliani ERJ, Aerts DRG C. Prevalência e determinantes de anemia em crianças de Porto Alegre, RS, Brasil. Rev Saúde Pública 2001; 35:66-73.

20. Secretaria de Estado da Saúde de Sergipe. III Pesquisa de Saúde Materno-Infantil e Nutrição do Estado de Sergipe: PESMISE/98. Brasília: Secretaria de Estado da Saúde de Sergipe/Escola de Nutrição, Universidade Federal da Bahia; 2001.

21. Neuman NA, Tanaka OY, Szarfarc SC, Guimarães PRV, Victora CG. Prevalência e fatores de risco para anemia no Sul do Brasil. Rev Saúde Pública 2000; 34:301-8.

22. Food and Agriculture Organization, United Nations/World Health Organization. Requirements of vitamin A, iron, folate and vitamin B12. Rome: Food and Agriculture Organization, United Nations/World Health; 1988. (Food and Nutrition Series, 23).

23. World Health Organization. Complementary feeding of young children in developing countries. A review of current scientific knowledge. Geneva: World Health Organization; 1998.

24. Monte CMG, Sá MLB. Guias alimentares para crianças de 6-23 meses no Nordeste do Brasil: da teoria à prática. Fortaleza: The British Council; 1998.
25. Farias Júnior G. Consumo alimentar de crianças menores de 5 anos no Estado de Pernambuco, 1997 [Dissertação de Mestrado]. Recife: Universidade Federal de Pernambuco; 2003.

26. Assis AMO, Barreto ML, Santos LMP, Sampaio LR, Magalhães LP, Prado MS, et al. Condições de vida, saúde e nutrição na infância em Salvador. Salvador: Escola de Nutrição/Instituto de Saúde Coletiva, Universidade Federal da Bahia; 2000.

27. Osório MM. Fatores determinantes da anemia em crianças. J Pediatr 2002; 78:269-78.

28. Szarfarc SC, Souza SB, Furumoto RAV, Brunken GS, Assis AMO, Gaudenzi EM, et al. Concentração de hemoglobina em crianças do nascimento até um ano de vida. Cad Saúde Pública 2004; 20:26674 .

29. Assis AMO, Gaudenzi EN, Gomes G, Ribeiro RC, Szarfarc SC, Souza SB. Níveis de hemoglobina, aleitamento materno e regime alimentar no primeiro ano de vida. Rev Saúde Pública 2004; 38:543-51.

30. Hurrel RF, Lynch SR, Trinidad TP, Dassenko SA, Cook JD. Iron absorption in humans as influenced by bovine milk proteins. Am J Clin Nutr 1989; 49:546-52.

31. Hallberg L, Rossander-Hultén L, Brune M, Gleerup A. Calcium and iron absorption: mechanism of action and nutritional importance. Eur J Clin $\mathrm{Nu}$ tr 1992; 46:317-27.

32. Ziegler EE, Jiang T, Romero E, Vinco A, Frantz JA, Nelson SE. Cow's milk and intestinal blood loss in late infancy. J Pediatr 1999; 135:720-6.

33. Jiang T, Jeter JM, Nelson SE, Ziegler EE. Intestinal blood loss during cow milk feeding in older infants: quantitative measurements. Arch Pediatr Adolesc Med 2000; 154:673-8.

Recebido em 08/Mar/2005

Versão final reapresentada em 13/Jan/2006 Aprovado em 07/Mar/2006 